Boletín de la Sociedad Zoológica del Uruguay, 2020

Vol. 29 (2): 150-159

ISSN 2393-6940

https://journal.szu.org.uy

\title{
ABUNDANCIA Y FENOLOGÍA DE ARTRÓPODOS DEPREDADORES EN SOJA: ANÁLISIS PRELIMINAR
}

\author{
Mariángeles Lacava ${ }^{1-4}$, Luis Fernando García ${ }^{2-4}$, Juan Pablo Burla ${ }^{3}$, Ramiro Tambasco ${ }^{4}$, Viviana \\ Franco $4-5$, Carmen Viera ${ }^{4-5}$. \\ 1 PDU Estudios interdisciplinarios de sistemas territoriales complejos, sede Rivera, CENUR Noreste, \\ Universidad de la República. \\ 2 CURE Treinta y Tres, UdelaR \\ ${ }^{3}$ CURE Rocha, UdelaR \\ 4 Ecología del Comportamiento, IIBCE, Ministerio de Educación y Cultura \\ 5 Entomología, Facultad de Ciencias, UdelaR
}

Autor para correspondencia: cviera@fcien.edu.uy

\section{RESUMEN}

Los artrópodos son organismos que se han adaptado a múltiples ecosistemas, incluyendo los agroecosistemas. La soja es un agroecosistema que ha sufrido una gran expansión y también la fauna acompañante. El conocimiento de esta fauna es escaso en Uruguay, fundamentalmente la de artrópodos depredadores, agentes de control biológico conservativo. En este trabajo se evaluó la fauna de depredadores de suelo en etapas fenológicas de germinación, primer nudo, floración, y llenado de grano. Se recolectó la fauna mediante trampas pitfall a lo largo y ancho del cultivo, y en zonas aledañas, con vegetación natural. Las familias predominantes fueron Lycosidae (Araneae), Carabidae (Coleoptera) y Formicidae (Hymenoptera). En general los índices de diversidad mostraron menores valores en la etapa de llenado de grano. Se encontró una interacción significativa entre el orden y la abundancia de artrópodos con una dominancia consistente de las arañas a lo largo de la mayoría de etapas fenológicas. En general, estos resultados preliminares, coinciden con otros estudios realizados a nivel regional. Se deberán muestrear otros sitios en diferentes zonas del país para evaluar si se mantiene la tendencia encontrada. Además, es necesario evaluar la eficacia de los depredadores encontrados sobre insectos-plaga de soja.

Palabras clave: artrópodos-depredadores, etapasfenológicas, soja, control-conservativo

\section{ABSTRACT}

Abundance and phenology of predatory arthropods in soybean: preliminary analysis. Arthropods are organisms that have been able to adapt to multiple ecosystems, including agroecosystems. Soybean is an agroecosystem that has undergone great expansion and also the accompanying fauna. The knowledge of this fauna is scarce in Uruguay, mainly for native predatory arthropods, agents of conservative biological control. In the present work, the fauna of soil predators is evaluated in the phenological stages of germination, first node, flowering, and grain filling. The fauna was collected by pitfall traps throughout the crop, and in surrounding areas, with natural vegetation. The predominant families were Lycosidae (Araneae), Carabidae (Coleoptera) and Formicidae (Hymenoptera). The diversity varied across phenological stages, being lower in the grain filling stage. We also found a significant interaction between the phenological stage, and arthropod order abundance, where spiders were one of the most abundant groups. Overall,these preliminary results agree with other studies carried out in soybean and other crops. Other sites in different areas of the country should be sampled to assess whether the trend found is maintained. Furthermore, it is necessary to evaluate together with the abundance the predatory efficacy of the groups in soybean insects-plague.

Keywords: arthropod-predators, phenological-stages, soy, conservative-control

\section{INTRODUCCIÓN}

La soja es uno de los cultivos más importantes en Uruguay (MGAP, 2017), el incremento en el área cultivada ha venido acompañado de un notable aumento en la importación y el uso de agroquímicos que son comúnmente empleados para el control de insectos considerados plagas potenciales (Catacore, 2012). Los productos utilizados en el control químico son insecticidas y herbicidas tradicionales como piretroides y glifosato. Pese a que tratar a los cultivos con estos productos es considerado de baja sostenibilidad, en Uruguay es un método ampliamente utilizado en la producción de soja actualmente (Soutullo et al., 2020). 
Una alternativa al control químico de plaga es el control biológico conservativo que se basa en el uso de enemigos naturales nativos presentes en cultivos, como posibles controladores de insectos fitófagos (Losey \& Denno, 1998). Esta estrategia de control biológico es sustentable en relación a otros tipos de manejo, como el químico y otros tipos de control biológico, porque promueve no sólo el control de plagas, sino que también promueve la conservación de fauna y flora nativa (Barbosa, 1998). En América Latina el interés en la sociedad por la conservación de la diversidad es cada vez mayor y va asociado a la disminución de utilización de químicos.

Los depredadores generalistas son un grupo relevante en el control biológico conservativo (Rutledge et al., 2004; Fiedler et al., 2008; Benamú et al., 2017). Ello se debe a que son capaces de consumir una amplia variedad de insectos entre ellos, los de interés económico en distintos cultivos (Kromp, 1999; Thomson, 1992; García et al., 2020). Dentro de los depredadores generalistas, las arañas y escarabajos de las familias Carabidae y Staphylinidae, son de los grupos más representativos, pues no sólo son muy diversos, sino también abundantes, en una amplia variedad de cultivos (Armendano \& González, 2010; Benamú et al., 2010). En el caso de las arañas, recientemente se ha demostrado que son capaces de reducir las poblaciones de plagas en una amplia variedad de cultivos (Michalko et al., 2019a; 2019b). Una tendencia similar ha sido observada en carábidos que pueden controlar de manera efectiva algunas especies de pulgones, larvas de insectos perjudiciales y gasterópodos (House \& Stinner, 1983). Las hormigas carnívoras, también han sido registradas como importantes agentes en la reducción de insectos herbívoros en cultivos (Choate \& Drummond, 2011)

En soja, Rypstra \& Carter (1995) demostraron que existe una correlación negativa entre la abundancia de arañas tejedoras y el daño efectuado por los insectos plaga, por lo tanto, la preservación de las arañas en este tipo de agroecosistema puede presentar grandes beneficios económicos. De manera similar Halaj et al. (2000), demostraron que ambientes enriquecidos en cultivos de soja promueven la presencia de arañas y carábidos, encontrándose una relación negativa entre la presencia de ambos grupos y el daño causado por las plagas sobre las plantas. Estos resultados sugieren el potencial de estos grupos como agentes de control biológico conservativo. En el caso de las hormigas, algunas especies de los géneros Solenopsis Westwood, 1840 y Pheidole Westwood, 1839, han sido consideradas como agentes reguladores de larvas de lepidópteros en cultivos de soja de los Estados Unidos (Way \& Khoo, 1992).

En cultivos de soja en Argentina y Paraguay, se han encontrado como grupos frecuentes de depredadores, varios grupos de hemípteros, arañas, dermápteros, coleópteros y neurópteros (Belorte et al., 2014; Liljesthröm et al., 2002; Martins et al., 2012; Perotti et al., 2014; Stepin et al., 2018). A pesar de la importancia de los artrópodos depredadores nativos en los cultivos de soja, son pocos los estudios en Uruguay en esta temática, donde se ha estudiado a los artrópodos como enemigos naturales en soja, grupos como los parasitoides y sus efectos sobre algunas plagas de la soja (Zerbino \& Panizzi, 2019).

Por lo tanto, teniendo en cuenta la importancia que estos organismos tienen en la regulación de artrópodos plaga, se pretende evaluar la composición en abundancia y diversidad de los artrópodos que se encuentran en los cultivos de soja, durante su desarrollo fenológico. Se pretende generar, además, una base de datos para llevar a cabo futuros estudios que evalúen el rol de los artrópodos depredadores como posibles agentes de control biológico conservativo en insectos-plaga de soja.

\section{MATERIAL Y MÉTODOS}

\section{Área de estudio}

La recolección de los individuos se realizó en un cultivo de soja de 40 hás, en el departamento de San José (34017'50.1" latitud sur y 57003'58.6" longitud oeste) sobre la ruta 11, aproximadamente a $10 \mathrm{Km}$ de la capital del Departamento, San José de Mayo.

\section{Método de recolección}

Se efectuaron recolectas en cuatro etapas fenológicas del cultivo propuestas por Fehr y Caviness (1979). Las etapas fueron germinación, primer nudo (V1), floración R1) y llenado de grano (R5). La selección de las etapas se basó en que aparecen diferentes artrópodos plaga, como himenópteros en la etapa de siembra, lepidópteros en la etapa de primer nudo y floración y hemípteros en la etapa de llenado de grano.

Los muestreos fueron realizados mediante trampas de caída (pitfall) que se ubicaron a lo largo y ancho del cultivo. Se diseñaron tres transectas dentro del cultivo dos en los bordes y una diagonal entre estas dos transectas. También se colocaron pitfall en una zona aledaña al cultivo con vegetación natural (Fig. 1). Se relevó un área total de $1500 \mathrm{~m}^{2}$. Las trampas fueron recipientes de $8 \mathrm{~cm}$ de diámetro en su boca por $15 \mathrm{~cm}$ de fondo (Brennan et al., 2005). Cada recipiente-trampa contenía dos terceras partes de agua, gotas de detergente, y una tercera parte de alcohol etílico al $70 \%$, el cual permite que los organismos que caigan, queden preservados (Curtis, 1980). El etanol fue utilizado teniendo en cuenta que permite la preservación de una amplia variedad de artrópodos (Hohbein \& Conway, 2018; Skvarla et al., 2014). Las trampas permanecieron activas siete días, teniendo en cuenta la alta tasa de evaporación del etanol. Las trampas se colocaron durante dos semanas consecutivas en cada una de las etapas fenológicas. Cada trampa se colocó con la boca a nivel del suelo, para interceptar artrópodos caminadores en los diferentes ambientes evaluados Los muestreos se realizaron entre diciembre de 2015 y marzo de 2016. 


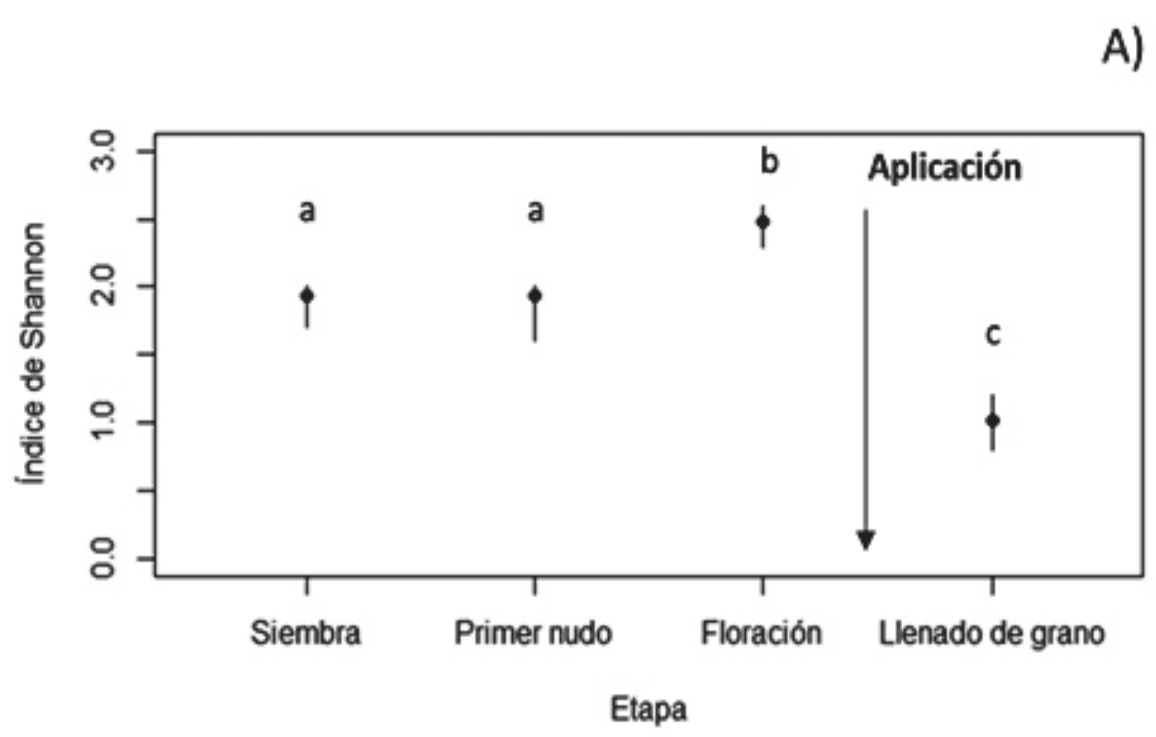

B)

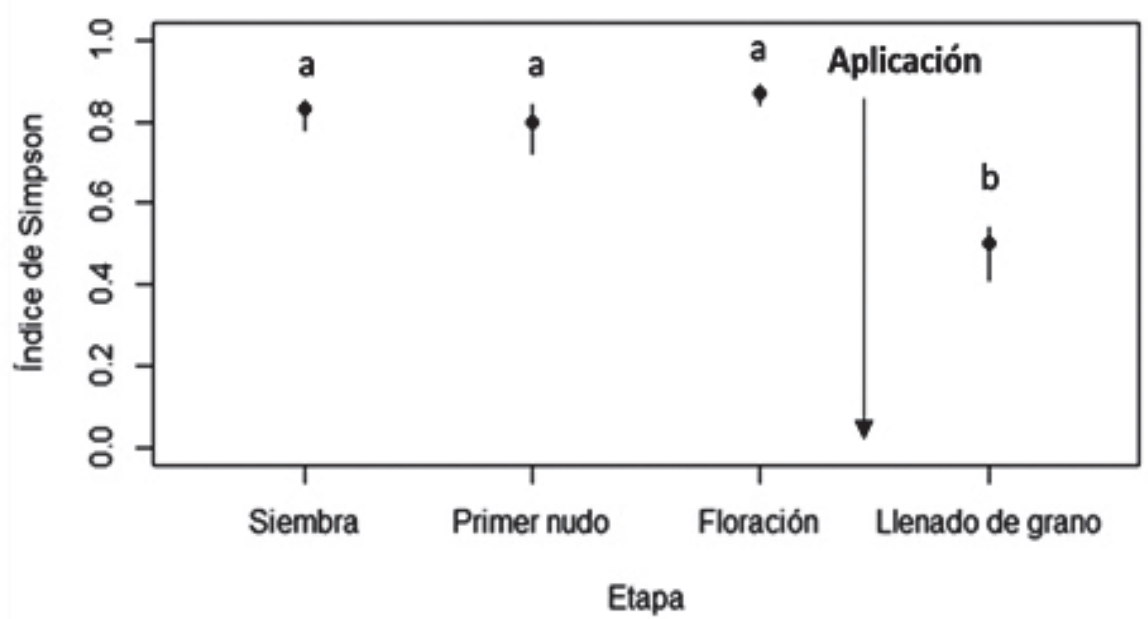

Fig. 1. Cambios en los índices de Shannon (A) y Simpson (B) en las diferentes etapas fenológicas del cultivo de soja. La flecha vertical indica la aplicación de insecticida. Los puntos indican los valores promedio del índice, las líneas indican los intervalos de confianza al 95\%, los parámetros fueron estimados mediante un análisis de Bootstrap.

Cada transecta dentro del cultivo tuvo $100 \mathrm{~m}$ de largo, con trampas cada 10 metros (10 trampas por transecta), salvo la diagonal que tuvo 8 trampas (Figura 1). En la zona aledaña se colocaron 20 trampas a una distancia de 10 metros, en dos transectas, completando 48 trampas. Los especímenes recolectados fueron llevados al laboratorio de Ecología del Comportamiento del Instituto de Investigaciones Biológicas Clemente Estable, e identificados mediante lupa estereoscópica, agrupados en familias y morfoespecies, utilizando para las arañas, la clave de Grismado et al. (2014). Los carábidos fueron identificados mediante la clave de Martínez (2003), mientras que las hormigas fueron identificadas con la clave de Palacio \& Fernández (2003).

Se determinó la eficiencia de muestreo, promediando la relación entre el número de especies observadas en relación a estimadores de abundancia (ACE, Chao 1) e incidencia (ICE; Chao2, Jackknife1, Jackknife 2 y Bootstrap) descritos para la evaluación de grupos similares en la región (Bragagnolo et al., 2003). La eficiencia del muestreo se estimó empleando el programa EstimateS (versión 910) (Colwell, 2013).

Una vez estimada la eficiencia del muestreo, se estimó y se comparó la diversidad utilizando los índices de Shannon-Wienner y de Simpson. Considerando que reflejan dos parámetros distintos de la diversidad, el 
Tabla 1. Lista de morfoespecies colectadas en el cultivo soja.

\begin{tabular}{cccc} 
Orden & Familia & Género & Especie \\
\hline Araneae & Linyphiidae & & Morfo sp. 1 \\
Araneae & Linyphiidae & & Morfo sp. 2 \\
Araneae & Linyphiidae & & Morfo sp. 3 \\
Araneae & Linyphiidae & & Morfo sp. 4 \\
Araneae & Lycosidae & Lycosa & Lycosa thorelli \\
Araneae & Lycosidae & Lycosa & Lycosa poliostoma \\
Araneae & Lycosidae & Lycosa & Lycosa cf. bivittata \\
Araneae & Tetragnathidae & Glenognatha & Morfo sp. 5 \\
Araneae & Thomisidae & & Morfo sp. 6 \\
Araneae & Theridiidae & & Morfo sp. 7 \\
Coleoptera & Staphylinidae & & Morfo sp. 8 \\
Coleoptera & Staphylinidae & & Morfo sp.9 \\
Coleoptera & Coccinellidae & & Morfo sp.10 \\
& & & \\
\hline
\end{tabular}

\begin{tabular}{cccc} 
Orden & Familia & Género & Especie \\
\hline Coleoptera & Coccinellidae & & Morfo sp.11 \\
Coleoptera & Carabidae & Pterospichus & Morfo sp.12 \\
Coleoptera & Carabidae & & Morfo sp. 13 \\
Coleoptera & Lampyridae & & Morfo sp. 14 \\
Hymenoptera & Formicidae & Camponotus & Morfo sp. 15 \\
Hymenoptera & Formicidae & & Morfo sp. 16 \\
Neuroptera & & & Morfo sp. 17 \\
Diptera & Dolichopodidae & & Morfo sp. 18 \\
Diptera & Dolichopodidae & & Morfo sp. 19 \\
Hemiptera & Reduviidae & & Morfo sp. 20 \\
Heteroptera & Nabidae & Nabis & Nabiscapsiformis \\
Heteroptea & Reduviidae & & Morfo sp. 21
\end{tabular}

primero es afectado por las especies raras, mientras que el segundo refleja la dominancia y es afectado por la abundancia de las especies más frecuentes (Pinkus Rendón et al., 2006), se usan ambos índices como parámetros frecuentes para evaluar la diversidad (Rubio \& Moreno, 2010; Privet \& Pétillon, 2020). El índice de Shannon se comparó entre las distintas etapas fenológicas mediante un análisis de Bootstrap con 100 iteraciones y un intervalo de confianza del 95\%, este análisis puede ser empleado para comparar los valores de los índices de diversidad cuando las réplicas son escasas (Gardener, 2014). El mismo procedimiento se realizó para comparar los valores del índice de Simpson entre las etapas fenológicas, debido a las comparaciones múltiples, en todos los casos la significancia fue ajustada mediante el método de Bonferroni. Los análisis fueron realizados en el programa estadístico R versión 3.6.3 (R Core Team, 2020).

Para establecer la existencia de interacción entre la etapa fenológica y los órdenes de artrópodos en el cultivo, se utilizó una ecuación de estimación generalizada con distribución Poisson, teniendo en cuenta que las trampas fueron utilizadas repetidamente a lo largo de las distintas etapas fenológicas del cultivo y este tipo (Zuur et al., 2009). Se consideraron como variables explicativas, la etapa fenológica, orden de artrópodos y lugar del muestreo (borde, zona aledaña o diagonal) y como variable aleatoria, el número de trampa.

\section{RESULTADOS}

Se recolectaron 454 ejemplares agrupados en seis órdenes, 16 familias y 25 morfoespecies depredadoras
(Tabla 1). En la evaluación de los valores de especies observadas en relación a los diferentes estimadores utilizados, se encontró una eficiencia de muestreo del $84 \%$.

Se encontraron diferencias significativas en la diversidad entre las diferentes etapas, con los dos índices de diversidad empleados. En el caso del índice de Shannon, la mayor diversidad se presentó en la etapa de floración, seguida por las etapas de siembra y formación del nudo que fueron similares entre sí (Fig. 1A). Los valores más bajos se presentaron a nivel de la etapa del llenado de grano. Según el índice de Simpson, todas las etapas fueron similares, a excepción del llenado de grano que presentó valores significativamente más bajos que el resto (Fig. 1B).

Al evaluar la abundancia de los diferentes órdenes de depredadores, se encontró que las arañas fueron el grupo más abundante, seguido por el orden Hymenoptera, representado exclusivamente por la familia Formicidae. El tercer orden más abundante fue Coleoptera. Los órdenes menos abundantes fueron Diptera, Hemiptera y Neuroptera respectivamente (Fig. 2). Dentro del orden Araneae, la familia más abundante fue Lycosidae, seguida por Linyphiidae, Tetragnathidae, Theridiidae y Thomisidae (Fig. 3A). En los coleópteros, la familia más abundante fue Carabidae seguida por Staphylinidae con una abundancia similar (Fig. 3B). En los himenópteros, la familia más abundante fue Formicidae, que también fue la más representativa respecto a los demás órdenes de insectos (Fig. 3C y Tabla 1).

Se encontró, además, un efecto significativo de la interacción entre las distintas etapas fenológicas del cultivo y la abundancia de los tres órdenes más 


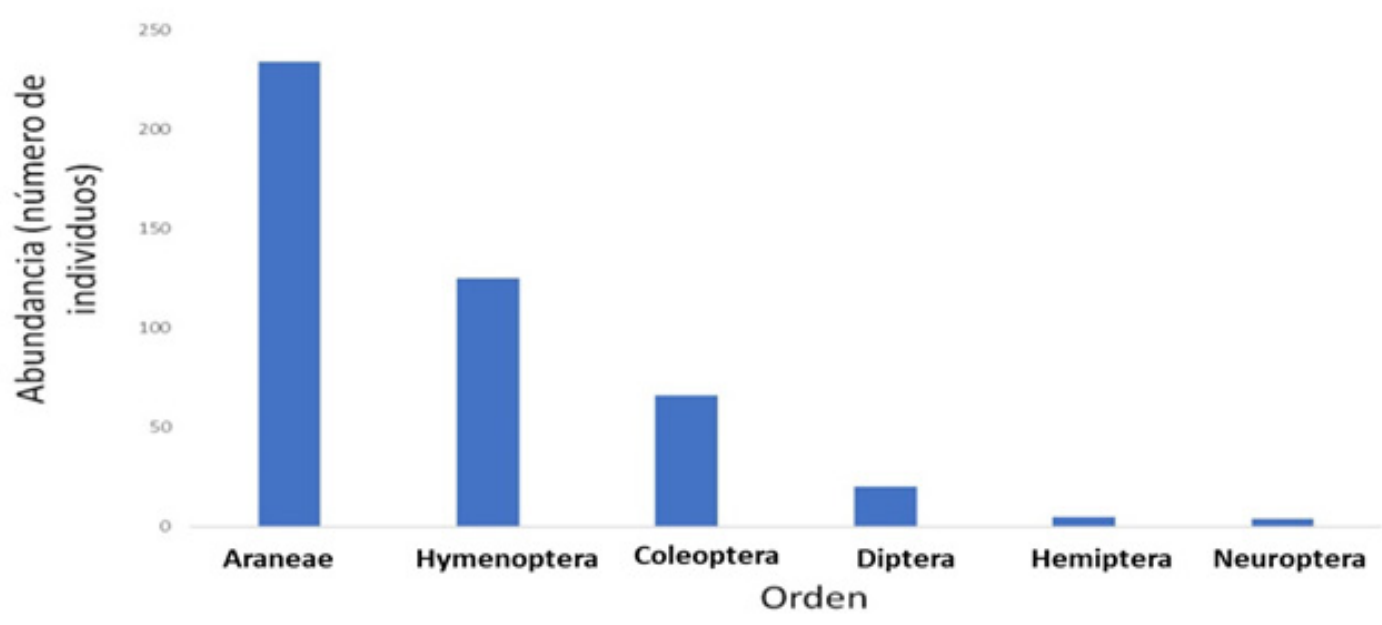

Fig. 2. Abundancia de los principales órdenes de artrópodos depredadores encontrados en el cultivo de soja.

representativos de artrópodos $\left(X^{2}=20.90, \mathrm{df}=6\right.$, $p<0.01)$. En general, las arañas fueron el grupo más abundante a lo largo de todas las etapas fenológicas del cultivo (Fig. 4). Las hormigas fueron el segundo grupo con mayor abundancia en todas las etapas, excepto la floración, donde fueron los coleópteros, este último grupo fue el tercero más abundante a lo largo de las restantes etapas de desarrollo. Los órdenes Diptera, Hemiptera y Neuroptera se encontraron únicamente en la etapa de floración.

\section{DISCUSIÓN}

Los muestreos presentaron una alta eficiencia colectando un promedio del $84 \%$ de las especies presentes en el cultivo, lo que sugiere que el número de trampas utilizadas fue adecuado, para colectar la diversidad presente, aunque estos resultados se limitan a una única localidad. A pesar de ello, la tendencia registrada en este estudio coincide con otros trabajos en otros cultivos, además de soja, donde se recomienda el uso de trampas de caída para colectar las especies de artrópodos de suelo (Knapp et al., 2020).

Al evaluar los resultados correspondientes a diversidad, se encontraron diferencias significativas en las diferentes etapas fenológicas del cultivo, para ambos índices utilizados. Con el índice de Shannon, la mayor diversidad se registró en la etapa de floración, un resultado esperable, teniendo en cuenta la mayor complejidad vegetal que se encuentra ligada con una mayor diversidad de varios grupos de artrópodos. Por ejemplo, se ha demostrado que una mayor complejidad estructural se relaciona con la diversidad en algunos grupos como las arañas (Lafage et al., 2019). Por otro lado, las etapas de siembra y formación del primer nudo fueron similares en cuanto a diversidad, debido posiblemente, a que las primeras etapas de la planta tienen un tamaño reducido y, en consecuencia, una complejidad estructural similar a la etapa de siembra. Finalmente, con el índice de Shannon, se observó un marcado descenso en la etapa de llenado de grano, lo cual puede deberse a la aplicación de insecticidas de amplio espectro usada por los productores, que tienen efectos conocidos en la diversidad de depredadores nativos (Epstein et al., 2000). A nivel del índice de Simpson, se observaron valores similares en las tres primeras etapas, sugiriendo una composición similar de la comunidad de depredadores, la cual muestra una caída marcada en la última etapa que también puede ser explicada por el uso de insecticidas. A pesar de lo anterior, estos resultados deben ser interpretados con cuidado debido a que se limitan a una única localidad.

A nivel de la abundancia de los grupos evaluados, los resultados de este trabajo coinciden con otros estudios en donde las arañas junto con los carábidos y hormigas carnívoras son los grupos más representativos de depredadores en los cultivos de soja (House \& Stinner, 1983). Aunque se esperaba una mayor abundancia para el caso de las hormigas, debido a los hábitos coloniales-sociales de algunas especies, no ocurrió, posiblemente debido a que las trampas pitfall utilizadas no presentaron atrayentes específicos para hormigas como cebos (FontalvoRodríguez \& Domínguez-Haydar, 2009), pudiendo haber sesgado la abundancia de este grupo. Aunque, las hormigas fueron el segundo grupo de 


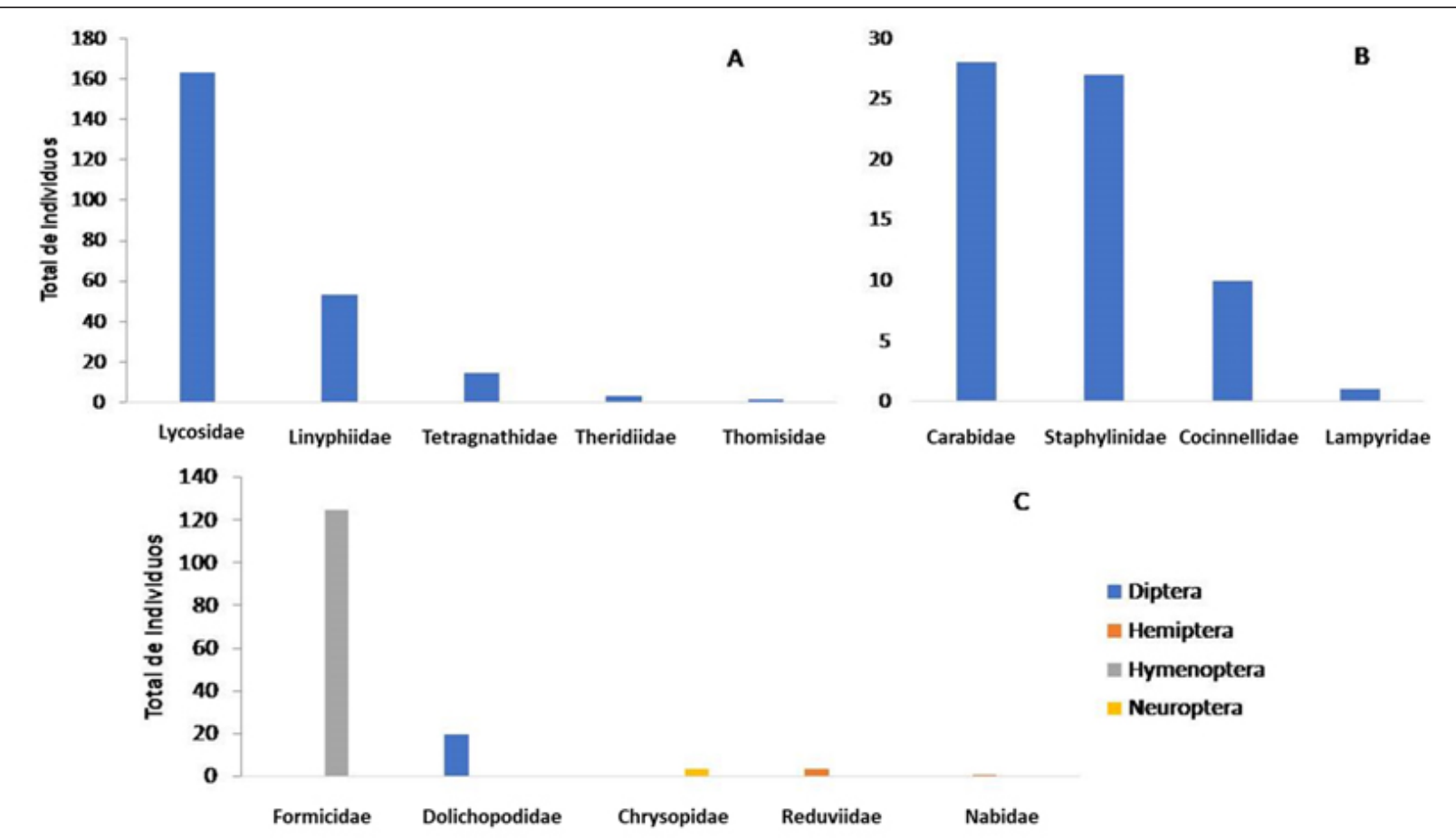

Fig. 3. Abundancia de A) familias de arañas, B) familias de escarabajos y C) familias de otros órdenes presentes en el cultivo de soja.

depredadores más abundantes después de las arañas, estos resultados deben ser interpretados cuidadosamente, considerando que las hormigas del género Camponotus Mayr, 1861 encontrado en el cultivo, puede presentar hábitos omnívoros o incluso promover el desarrollo de algunas plagas, además de las especies con hábitos depredadores (RicoGray \& da Silveira Lobo Sterberg, 1991). A nivel regional, no hay estudios relacionando las interacciones entre arañas y hormigas en cultivos de soja. Sin embargo, estudios realizados sobre la diversidad de artrópodos depredadores en Indonesia, han encontrado tendencias opuestas a las de este estudio, con una mayor abundancia de hormigas, seguida por las arañas (House \& Stinner, 1983). Posiblemente las diferencias puedan explicarse debido a la diferencia en los métodos de colecta utilizados, así como a la mayor diversidad de algunos grupos, como las hormigas en zonas tropicales (Piper et al., 2009).

En el caso de las arañas, la marcada abundancia de este grupo, coincide con otros estudios, realizados en otros cultivos de la región Armendano \& González, 2010; Almada et al., 2016; Benamú et al., 2017). A nivel de las familias evaluadas, Lycosidae fue la más abundante, debido a sus hábitos cursoriales que incrementan la probabilidad de caída en las trampas
(Willemart \& Lacava, 2017). La abundancia de arañas lobo estuvo seguida por la familia Linyphiidae, que, a pesar de ser arañas constructoras de tela, frecuentemente son capturadas en trampas de caída (Topping \& Luff, 1995). Lycosidae y Linyphiidae son arañas con mucha tolerancia a los métodos de cultivo, por lo tanto aparecen como fauna nativa en agroecosistemas, lo que explica su marcada abundancia en este cultivo (Ferguson et al., 1984), así como en otros cultivos de la región (Almada et al., 2016; Armendano \& González, 2010; Benamú et al., 2017), Estados Unidos y Europa (Nyffeler et al., 2003). Otras familias como Thomisidae, Tetragnathidae y Theridiidae, si bien han sido reportadas como grupos importantes en el cultivo de soja (Meena \& Sharma, 2019), su baja abundancia pueda deberse a que se encuentran principalmente encima de la vegetación (Cardoso et al., 2010; Meena \& Sharma, 2019), por lo que las trampas de caída pueden haber sido poco efectivas para capturar estas arañas.

Los carábidos y estafilínidos fueron los grupos más representativos dentro del orden Coleoptera, y posiblemente caen frecuentemente en trampas pitfall, debido a sus hábitos cursoriales (Martins et al., 2012). Como en el caso de las arañas de las familias Lycosidae y Linyphiidae, los carábidos y estafilínidos son considerados organismos capaces de soportar 

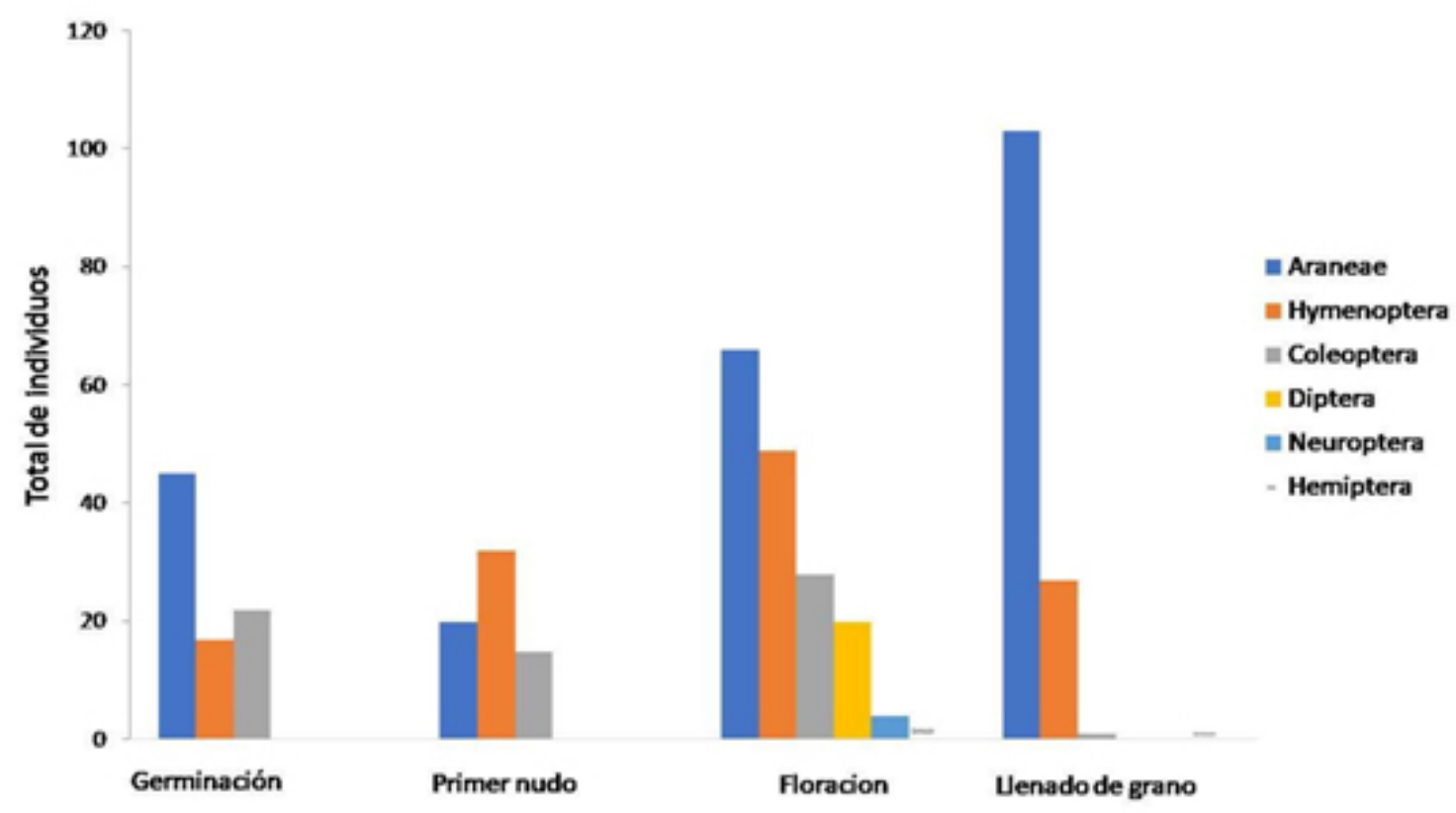

Fig. 4. Abundancia de los principales órdenes de artrópodos depredadores presentes a lo largo de las distintas etapas fenológicas del cultivo.

las perturbaciones generadas por actividades antrópicas en el cultivo de soja (Brunke et al., 2014). Estos resultados coinciden con otros estudios de diversidad de depredadores en cultivos de soja, dónde las familias Carabidae y Staphylinidae son los grupos más abundantes en los cultivos de soja. La menor abundancia de la familia Coccinellidae, puede deberse al hecho de que los escarabajos de esta familia se encuentran principalmente en el follaje (Cividanes et al., 2009), por lo que las trampas de caída capturaron estos insectos en bajas proporciones. Una tendencia similar puede haberse presentado en los demás grupos de insectos, principalmente los hemípteros y neurópteros que son grupos de depredadores representativos de soja y que pueden haber presentado bajas densidades, debido a que presentan fundamentalmente hábitos foliares. Se han registrado dípteros de la familia Dolichopodidae en las trampas de caída, pese a no ser el mecanismo de colecta más eficiente para este grupo (Pollet \& Grootaert, 1987).

La interacción significativa entre los órdenes y las etapas fenológicas del cultivo en relación a la abundancia de los diferentes grupos, fueron fundamentalmente ocasionadas por las abundancias de arañas y hormigas en las etapas de germinación y primer nudo, donde ambos grupos presentaron patrones inversos. Estos resultados podrían explicarse por la aparición de plántulas, que permiten la llegada de nuevas presas que incrementan la actividad de las hormigas dentro del cultivo. Las arañas y las hormigas pueden presentar abundancias inversas debido a la competencia (Wise, 1995). Dicha competencia puede verse intensificada en la etapa de plántula de la soja debido a su menor complejidad estructural. La mayor abundancia de arañas en etapas posteriores en relación a otros depredadores, puede deberse a la mayor complejidad estructural de la planta que permite el establecimiento de distintos gremios (Greenstone, 1984). Adicionalmente un posible incremento en la disponibilidad de presas que acompañe el desarrollo de la planta, puede reducir la competencia entre las arañas y hormigas disponibles en el cultivo, algunos estudios, por ejemplo, han demostrado que una mayor complejidad estructural en los cultivos se relaciona con una mayor abundancia de presas disponibles para las arañas (Diehl et al., 2013). Futuros estudios deberían enfocarse en aspectos como la competencia inter-específica entre arañas y hormigas. Por otra parte la abundancia de escarabajos que presentó un marcado descenso en la etapa del llenado de grano posiblemente puede ser explicada porque coincidió con la aplicación de insecticidas (piretroides), los cuales son particularmente severos contra este grupo de organismos como se ha demostrado en otros estudios (Pekár, 2012; Pisa et al., 2014; Tooming et al., 2017; Vickerman et al., 1987).

Los resultados obtenidos en este estudio preliminar, realizado en un predio, coinciden en general con otros 
estudios realizados regionales en otros cultivos y Norteamérica. Futuros estudios deberían enfocarse en evaluar si la misma tendencia encontrada en los cultivos evaluados se presenta en cultivos de soja de otras zonas del país. Es necesario analizar el papel ecológico de los depredadores registrados en este estudio sobre las plagas locales, ya que algunos grupos como arañas Lycosidae ya han sido reportados como posibles depredadores de plagas locales como chinches y lagartas (García et al., en prensa). El mismo servicio ecosistémico sería esperable para otros depredadores encontrados, como coleópteros y hormigas.

\section{AGRADECIMIENTOS}

Queremos agradecer a la Comisión Sectorial De Investigación Científica (CSIC)por la financiación del proyecto y a Ramiro Tambasco por su ayuda en las salidas de campo. Alexánder Sabogal amablemente colaboró con la discusión relacionada con los índices de diversidad. Los revisores anónimos contribuyeron con sus sugerencias al mejoramiento del trabajo

\section{BIBLIOGRAFÍA}

Almada M., González, A. \& J. Corronca. 2016. Cambios en la comunidad de arañas (Arachnida: Araneae) en períodos de barbecho y de cultivos de soja en el Norte de Santa Fe, Argentina. Revista de la Facultad de Agronomía Universidad de la Plata, 115: 55-65.

Armendano A. \& A. González. 2010. Comunidad de arañas (Arachnida, Araneae) del cultivo de alfalfa (Medicago sativa) en Buenos Aires, Argentina. Revista de Biología Tropical, 58: 757-767.

Barbosa P.A. 1998. Conservation biological control. Elsevier. 396 pp

Belorte L.C.C., Ramir Z.A. \& A.M. Faria. 2004. Ocorrência de predadores em cinco cultivares de soja [Glycinemax (I.) merrill, 1917] no município de Araçatuba, sp.Arq. Inst. Biol., São Paulo, 71:45-49.

Benamú M.A., Schneider M.J. \& N.E. Sánchez. 2010. Effects of herbicide glyphosate on biologivl attributes of Alpaida veniliae (Araneae, Araneidae), in laboratory. Chenosphere, Doi 10.1016/j.chemosphere.2009.11.027.

Benamú M.A., Lacava M., García L.F., Santana M. \& C. Viera. 2017. Spider associated with agroecosystems: roles and perspectives. En: Viera C. \& Gonzaga M. (Eds.). Behaviour and Ecology of Spiders. Contributions from the Neotropical Region, pp. 275-303. Springer.

Bohan D.A., Boursault A., Brooks D.R. \& S. Petit. 2011. National-scale regulation of the weed seedbank by carabid predators. Journal of Applied Ecology, 48: 888-898.
Bragagnolo C. \& R. Pinto-da-Rocha. 2003. Diversidade de opiliões do parque nacional da serra dos órgãos, Rio de Janeiro, Brasil (Arachnida: Opiliones). Biota Neotropica, 3:120.

Brennan K.E.C., Majer J.D. \& Moir M.L. 2005. Refining sampling protocols for inventorying invertebrate biodiversity: influence of drift-fence length and pitfall trap diameter on spiders. The Journal of Arachnology, 33(3):681-702.

Brunke A., Klimaszewski B. \& R. Hallett. 2014. Rove beetles (Coleoptera: Staphylinidae) in Ontario, Canada soybean agroecosystems: Assemblage diversity, composition, seasonality, and habitat use. The Canadian Entomologist, 146: 1-19. 10.4039/tce.2014.19.

Bugg R. L., Dutcher J.D. \& P.J. McNeill. 1991. Coolseason cover crops in the pecan orchard understory: effects on Coccinellidae (Coleoptera) and pecan aphids (Homoptera: Aphididae). Biological Control, 1(1), 8-15.

Catacore G., Galeano P., Afapito-Tenfer S.Z. \& R Nodari. 2012. Soybean production in the southern cone of Americas: update on land and pesticide use. Technical Report, DOI 10.13140/ 2.1.1064.4804.

Colwell R.K. 2013. EstimateS: Statistical estimation of species richness and shared species from samples. Journal Published at: http:// purl.oclc.org/estimates.

Choate B. \& F. Drummond. 2011. Ants as biological control agents in agricultural cropping systems. Terrestrial Arthropod Reviews, 4 :157180

Cividanes F.J., Barbosa J.C., Ide S., Perioto, N.W. \& R. Lara. 2009. Faunistic analysis of Carabidae and Staphylinidae (Coleoptera) in five agroecosystems in northeastern São Paulo state, Brazil. Pesquisa Agropecuária Brasileira, 44: 954-958.

Curtis D.J. 1980. Pitfalls in spider community studies (Arachnida, Araneae). Journal of Arachnology, 8: 271-280.

Diehl E., Mader V., Walter V. \& K. Birkhofer. 2013. Management intensity and vegetation complexity affect web-building spiders and their prey. Oecologia, 173: 579-589.

Epstein D.L., Zack J.F., Brunner L., Gut J. \& J. Brown. 2000. Effects of broad-spectrum insecticides on epigeal arthropod biodiversity in Pacific Northwest Apple Orchards. Environmental Entomology, 29 (2): 340-358.

Fehr W. R. \& C. Caviness. 1979. Stages of soybean development. Special Report of Cooperative Extension Service Agriculture and Home Economic Experiment. Station lowa Publication: 1-13.

Ferguson H.J., McPherson R.M. \& W.A. Allen. 1984. Arañas que viven en el suelo y en el follaje en 
cuatro sistemas de cultivo de soja. Entomología ambiental, 13: 975- 980.

Fiedler A.K., Landis D.A., \& Wratten S.D. 2008. Maximizing ecosystem services from conservation biological control: the role of habitat management. Biological control, 45: 254-271.

Fontalvo-Rodríguez L. \& Y. Domínguez-Haydar. 2009. Ectatomma ruidum (Roger) como indicadora de diversidad de hormigas cazadoras (Hymenoptera: Formicidae) y relación con estructura vegetal en parches de bosque seco del Caribe colombiano. Intropica, 29-39.

García L.F., Núñez E., Lacava M., Silva H. \& J. Pétillon. 2020. Experimental assessment of trophic ecology in a generalist spider predator: Implications for biocontrol in Uruguayan crops. Journal of Applied Entomology, DOI: 10.1111/ jen.12811

Gardener M. 2014. Community Ecology: Analytical methods using $R$ and Excel. Pelagic Publishing. $556 \mathrm{pp}$.

Greenstone M.H. 1984. Determinants of web spider species diversity: vegetation structural diversity vs. prey availability. Oecologia, 62: 299-304.

Grismado C.J., Ramírez M. \& M. Izquierdo. 2014. Araneae: taxonomía, diversidad y clave de identificación de familias de la Argentina. Biodiversidad de Artrópodos Argentinos, 3: 55 -94

Halaj J. Cady C.A. \& G.W. Uetz. 2000. Modular Habitat Refugia Enhance Generalist Predators and Lower Plant Damage in Soybeans. Environmental Entomology, 29:383-393.

Hohbein R.R. \& C.J. Conway. 2018. Pitfall traps: A review of methods for estimating arthropod abundance. Wildlife Society Bulletin, 42 (4): 597-606.

House G.J. \& B.R. Stinner. 1983. Arthropods in notillage soybean agroecosystems: community composition and ecosystem interactions. Environmental Management, 7: 23-28.

Knapp M., Knappová J., Jakubec P., Voni ka P. \& P. Moravec. 2020. Incomplete species lists produced by pitfall trapping: How many carabid species and which functional traits are missing? Biological Conservation, 245: 108-545.

Kromp B. 1999. Carabid beetles in sustainable agriculture: a review on pest control efficacy, cultivation impacts and enhancement. Agriculture, Ecosystems \& Environment, 74: 187-228.

Lafage D., Djoudi E.A., Perrin G., Gallet S. \& J. Pétillon. 2019. Responses of ground-dwelling spiders assemblage to changes in vegetation from wet oligotrophic habitats of western France. In: Arthropod-plant interactions, 13: 653-662.

Liljesthrom G., Minervino E., Castro D. \& A. Gonzalez. 2002. La comunidad de arañas del cultivo de soja en la provincia de Buenos Aires, Argentina. Neotrop. Entomol., 31(2): 197-209.

Losey J.E. \& R.F. Denno. 1998. Positive predator- predator interactions: enhanced predation rates and synergistic suppression of aphid populations. Ecology, 79: 2143-2152

Martínez C. 2005. Introducción a los escarabajos Carabidae (Coleoptera) de Colombia. Instituto de Investigación de recursos biológicos Alexander von Humboldt: 1-546.

Martins I.C.F., Cividanes F.J. \& G.Q. Haddad. 2012. Diversidade e preferência de habitat de Carabidae e Staphylinidae (Coleoptera) em dois agroecossistemas. Bragantia, 71:471-480.

Meena L.K. \& A.N. Sharma. 2019. Biodiversity, guild structure and vertical stratification of spiders in soybean ecosystem. Journal of Entomology and Zoology Studies, 7: 1002-1004.

MGAP (Ministerio de Agricultura, Ganadería y Pesca). 2017. Anuario Estadístico Agropecuario.(http:// https://www.gub.uy/ministerio-ganaderiaagricultura-pesca/comunicacion/publicaciones/ anuario-estadistico-diea-2017). Acceso mayo 2020.

Michalko R., Pekár S. \& M. Entling. 2019a. An updated perspective on spiders as generalist predators in biological control. Oecologia, 189: 21-36.

Michalko R., Pekár S., Dulá M. \& M.H. Entling. 2019b. Global patterns in the biocontrol efficacy of spiders: A metaanalysis. Global Ecology and Biogeography, 28:1366-1378.

Nyffeler M. \& K.D. Sunderland. 2003. Composition, abundance and pest control potential of spider communities in agroecosystems: A comparison of European and US studies. Agriculture Ecosystems and Environment, 95: 579-612.

Palacio E.E. \& F. Fernández. 2003. Clave para las subfamilias y géneros, cap. 15. En: Fernández F. (Ed.). Introducción a las hormigas de la región Neotropical, pp. 233-261. Instituto de Investigaciones de Recursos Biológicos, Alexander von Humboldt.

Perotti E., Russo R., Lopez R., Zari F., Pradolini E., Sanmarti N., Maccari G., Boero L \& J. Gamundi. 2014. Evaluación de estrategias de manejo de refugios asociados a cultivos de soja Bt sobre plagas blanco, no blanco y sus depredadores. Revista para mejorar la producción 52-INTA EEA Oliveros, 177-182.

Pekár S. 2012. Spiders (Araneae) in the pesticide world: an ecotoxicological review. Pest Management Science, 68: 1438-1446. https:// doi.org/10.1002/ps.3397

Pinkus Rendón M.A., Ibarra-Núñez G., Parra-Tabla V., García - Ballinas J.A. \& Y. Hénaut. 2006. Spider diversity in coffee plantation with different management in Southeast Mexico. Journal of Arachnology, 34 (1): 104-112.

Piper S.D., Catterall C.P., Kanowski J.J. \& H.C. Proctor. 2009. Biodiversity recovery during rainforest reforestation as indicated by rapid assessment of epigaeic ants in tropical and subtropical 
Australia. Austral Ecology, 34: 422-434.

Pisa L.W., Amaral-Rogers V., Belzunces L.P., Bonmatin J.M., Downs C.A., Goulson D., Kreutzweiser D.P., Krupke C., Liess M., Mcfield M., Morrissey C.A., Noome D.A., Settele J., Simon-Delso N., Stark J.D., Van Der Sluijs J.P., Van Dyck H. \& M. Wiemers. 2014. Effects of neonicotinoids and fipronil on non-target invertebrates. Environmental Science and Pollution Research, 22: 68-102.

Pollet M. \& P Grootaert. 1987. Ecological data on Dolichopodidae (Diptera) from a woodland ecosystem: I. Colour preference, detailed distribution and comparison of different sampling techniques. Bull. Inst. R. Sc. Nat. Belg.(Entomol.), 57: 173-186.

Privet K.L. \& J. Pétillon. 2020. Comparative pattern in taxonomic and functional spider diversity between tropical vs temperate forest. Ecology and Evolution, Doi.org/1002/ece3.6907.

R Core Team. 2020. R: A language and environment for statistical computing. R Foundation for Statistical Computing, Vienna, Austria. URL https://www.R-project.org/.

Rico-Gray V. \& L. Da Silveira Lobo Sterberg. 1991. Carbon isotopic evidence for seasonal change in feeding habits of Camponotus planatus Roger (Formicidae) in Yucatán, Mexico. Biotropica, 23 (1): 93-95.

Rubio G.D. \& C. Moreno C.E. 2010. Orb-weaving spider diversity in the Iberá Marshlands, Argentina. Neotropical Entomology, 39:496-505.

Rutledge C.E., O Neil R.J., Fox T.B. \& D.A. Landis. 2004. Soybean aphid predators and their use in integrated pest management. Annals of the Entomological Society of America, 97: 240-248.

Rypstra A.L. \& P.E. Carter. 1995. The Web-Spider Community of Soybean Agroecosystems in Southwestern Ohio. The Journal of Arachnology, 23: 135-144.

Skvarla M.J., Larson J.L. \& A.P.G. Dowling. 2014.Pitfall and preservative: a review. Journal of Entomological Society of Ontario, 145:15-43.

Soutullo A., Ríos M., Zaldúa N. \&Teixeira-De-Mello F. 2020. Soybean expansion and the challenge of the coexistence of agribusiness with local production and conservation initiatives: Pesticides in a Ramsar site in Uruguay.
Environmental Conservation, 47:97-103.

Stepin-Sytnik E.V., Garay-Villalba M.C., MaidanaOjeda M., Cabral-Antúnez C. 2018. Insectos depredadores y parasitoides asociados al cultivo de soya (Glycinemax L. Merrill, 1917), en el distrito Arquitecto Tomás Romero Pereira, Itapúa, Paraguay. Intropica, 13:122129.

Thomson W.T. 1992. A worldwide guide to beneficial animals (insects/mites/nematodes) used for pest control purposes. Thomson Publications. $91 \mathrm{pp}$.

Tooming E., Merivee E., Must A., Merivee M.I., Sibul I., Nurme K. \& I.H. Williams. 2017. Behavioural effects of the neonicotinoid insecticide thiamethoxam on the predatory insect Platynusassimilis. Ecotoxicology, 26: 902-913.

Topping C. \& M. Luff. 1995. Three factors affecting the pitfall trap catch of linyphiid spiders (Araneae: Linyphiidae). Bulletin of the British Arachnological Society, 10. 35-38.

Vickerman G.P., Coombes D.S., Turner G., MeadBriggs M.A. \& J. Edwards. 1987. The effects of pirimicarb, dimethoate and deltamethrin on Carabidae and Staphylinidae in winter wheat. The effects of pirimicarb, dimethoate and deltamethrin on Carabidae and Staphylinidae in winter wheat, 52:213-223.

Way M.J. \& K.C. Khoo. 1992. Role for ants in pest Management. Annu. Rev. Entomol., 37:479-503

Willemart R.H. \& M. Lacava. 2017. Foraging strategies of cursorial and ambushing spiders. En: Viera C. \& Gonzaga M. (Eds.) Behaviour and Ecology of Spiders: Contributions from the neotropical region, pp. 227-247. Springer.

Wise D.H. 1995. Spiders in ecological webs. Cambridge University Press.

Zerbino M.S. \& A.R. Panizzi. 2019. The underestimated role of pest pentatomid parasitoids in Southern South America. Arthropod-Plant Interactions, DOI: 10.1007/s11829-019-09703-1.

Zuur A., Leno E.N., Walker N., Saveliev A.A. \& G.M. Smith. 2009. Mixed effects models and extensions in ecology with R. Springer Science \& Business Media.

Fecha de Recepción: 12 de septiembre de 2020 Fecha de Aceptación: 17 de diciembre 2020 